\title{
Application of Pattern Language for Game Design in Pedagogy and Design Practice
}

\author{
Christopher Aaron Barney ${ }^{*}$
}

1 Northeastern University; c.barney@northeastern.edu

* Correspondence: c.barney@northeastern.edu; Tel.: +1 (671) 939-7868

\begin{abstract}
Existing implementations of game design patterns have largely been confined to theoretical or research settings. Weaknesses in these implementations have prevented game design patterns from being properly evaluated as an educational and practical development tool. This paper examines these weaknesses, describes a method of developing and applying patterns that overcome the weaknesses, and evaluates use of the method for game design education and practice.

Weaknesses in existing pattern implementations are: omission of design problems, presumption of functional completeness at the level of pattern languages, narrow topical focus, and lack of a concise, repeatable method for pattern production. Several features of the proposed method were specifically built to address these weaknesses, namely the pattern template, the process for connecting patterns into a language and assessing the language's scope, a rubric for assessing pattern confidence and interconnectivity confidence, and pattern-building exercises.
\end{abstract}

This method was applied in a classroom setting. Results as assessed by the evaluation of student work suggest that creating patterns/pattern languages is an effective pedagogical approach. Designs produced using designer-created patterns closely align with existing design theory and are clearly understood by students.

The above results may indicate that the path to gaining wider acceptance of pattern theory as a design framework within game design is not to produce a universal pattern language but to facilitate the creation of case-specific languages, by students and professional designers, that use a shared ontology and thus can be combined easily to solve the diverse sets of problems faced by these groups.

Keywords: game design, design patterns; pattern language; design pattern application; design pattern creation

\section{Introduction}

Game design as an industry operates under a significant number of constraints, from financial to labor to audience. Seeking optimal solutions, it operates in a constant state of self-definition. Game design as an academic discipline is likewise in a state of rapid change as it tries to balance a pursuit of understanding of the discipline as manifest in the products of the industry and the more measured development of theory and practice based on the research interests of scholars.

The problem of how to study, teach and practice game design has proven to be nontrivial. Practically these three goals have been pursued independently with varying degrees of success. Profound games such as Train [25] or This War of Mine [31] have been 
made as well as financially successful ones such as Call of Duty [9] or Fortnight [13]; occasionally these two coincide as in the case of Spec Ops: the Line. [28] Insightful studies are conducted regularly and game scholars leverage their design insights to produce serious games for educational, research, and training purposes. Games Studies programs such as those at Full Sail University and DigiPen Institute of Technology have been developed to help train students as practical developers and others such as the Game Science and Design program at Northeastern University have been created to train students as games researchers. Broadly though, these three areas of development have failed to find a shared ontology that allows advances in one to be readily applied to the others. Frameworks such as Mechanics Dynamics and Aesthetics (MDA) [17], Four Keys to Fun [19], The Five Domains of Play [30] or Jessie Schell's Design Lenses [26] have proven very useful. However none of these specific attempts at creating a framework addresses the full scope of game design. [10][22] Each has been met with resistance by some of the proponents of the others.

Researchers, educators, and game designers have failed to agree on a shared ontology because they have divergent needs. Even within the game development industry, the needs of one set of developers differ radically from those of another to the degree that having a shared design vocabulary can seem unproductive.

This paper argues that the solution is not to produce a universal framework but to develop case-specific frameworks that use a shared ontology and thus can be combined easily to solve the diverse sets of problems faced by these groups. The solution can be implemented by applying the idea of design patterns and pattern languages to the game design space.

\section{What is a design pattern?}

The concept of a design pattern was introduced in 1977 by Christopher Alexander as a solution to problems he saw in architectural design. He defines an architectural pattern as "a careful description of a perennial solution to a recurring problem within a building context, describing one of the configurations that brings life to a building. Each pattern describes a problem that occurs over and over again in our environment, and then describes the core solution to that problem, in such a way that you can use the solution a million times over, without ever doing it the same way twice." [2]

The use of patterns has developed across disciplines over the intervening forty years and will be discussed in the next section. The core definition of a pattern has been consistent throughout this period of development, though different implementations have emphasized or minimized aspects of Alexander's initial concept.

A brief history of pattern development and use

Alexander's book A Pattern Language: Towns, Buildings, Construction articulated his rationale for creating design patterns related to architecture. This book detailed 253 patterns and linked them together into a loose hierarchical language. The specifics of this work as it relates to game design patterns are discussed in more detail in Pattern Language for Game Design. [4]

Some time after the publication of A Pattern Language Alexander's work attracted the attention of computer scientists. By the 1990's a group of four scholars had adapted his ideas for use in object oriented programming in the book Design Patterns: Elements of Reusable Object-Oriented Software. [16] This book defined 23 patterns for use in this domain. The patterns produced by this group clarified thinking around object oriented 
programming and became a central organizing force in the practice for the decades since their publication. This effect demonstrates the utility of pattern theory. However, this limited set of patterns was seen as sufficient, and likely compete. Additionally, these patterns focused on the details of each solution and largely omitted the specific design problems that the patterns solved. Lastly the focus of this small language was in object oriented programming, that is in and of itself reasonable. However, many programmers saw the utility of these patterns and used it as an argument for the exclusiveness of the object oriented paradigm. These three shortcomings in the language, rather than in the validity of the patterns, have occurred to a greater or lesser degree in many of the pattern subsequent language applications.

In the four decades since the publication of Alexander's originating work patterns have spread to a number of other fields, the most notable being social and behavioral science. In particular the project by Takashi Iba to develop a pattern language for creative learners describes his large-scale project to develop a pattern language. [18] This project is notable in that while it does propose a fixed language it also clearly describes a repeatable process that was followed to create the language.

\section{Pattern projects in games}

Existing general pattern languages show some of the methodological shortcomings discussed in the previous section; presumed completeness, omission of design problems, or undeclared narrowness of focus. Examples are the two seminal pattern languages in games created by Adams and Dormans and by Björk and Holopainen respectively.

In the book Game Mechanics: Advanced Game Design [1], the authors created a small pattern library that was narrowly focused on game systems mechanics. The versatility and atomic nature of their patterns was very impressive. However, their work was similar to that of Gamma et. al. in that the small library was considered to be functionally complete. This limits the ability to directly connect patterns from this library with those from different areas of design or to understand how patterns from these different areas might interact with those from this library. It also introduces the risk of privileging those aspects of mechanical design over other parts of the discipline.

The scholars Stafan Björk and Jussi Holopainen first published a paper titled Patterns in Game Design [7] and later a book titled Game Design Patterns [6] where they began to produce a large pattern library. This library shifts focus heavily to the pattern and away from the problem that the pattern addresses. The library of patterns produced by this work has grown to include more than 700 patterns. [5] Unlike previously discussed languages, it is generalized and covers a broad section of the field. It is also not considered complete and continues to grow over time. However, the breadth and depth of this repository can limit its usefulness to scholars and developers who have not been part of its production. The scale of the library exacerbates the problem created by omitting design problems, and results in difficulty understanding what patterns are applicable under what circumstances. Ongoing work on this project is beginning to address this problem using visualization techniques to improve discoverability. [21]

There are a significant number of other game design related projects and I am limiting my discussion here to these two as they have had the broadest impact. However, the vast majority of existing projects have at least one of the above faults: narrow focus, presumed completeness, or failing to state the design problem addressed by each pattern. 


\section{Using patterns in pedagogy}

An interesting use of patterns as part of creating a pedagogy can be seen in a set of papers by researchers of serious games for education. The first of these is Design Patterns for Learning Games [19] Proposes combining the use of game design patterns drawn from the library created by Björk and Holopainen, pedagogical patterns from the Pedagogical Patterns Project [11] and the twenty two learning and teaching functions proposed by Shuell and Moran [27] to create games that effectively teach a particular knowledge set. These ideas are further developed and tested in the papers Game Design Techniques for Software Engineering Management Education [27] and Teaching Software Engineering Topics Through Pedagogical Game Design Patterns: An Empirical Study. [12] The results of the studies indicate that the use of patterns is effective in designing games that effectively teach specific subject matter. This work differs from this project in that the patterns being used are static and drawn from an external source, and that the source of learning is the produced game artifact. Whereas the process discussed in this paper uses the development of patterns to teach the discipline of game design as well as the use of patterns to produce games with intended effects.

The process for creating and using patterns described in this paper was created independently from the above line of research. However, the results section of this paper provides an assessment of the process I introduce here in light of the twenty two learning and teaching functions described by Shuell and Moran, and creates a mapping to the process used for pattern creation discussed in this paper. This full mapping is included as supplementary material.

\section{An alternative application of patterns}

The application of patterns as described in this paper arose out of a practice-driven application of pattern theory in the classroom and as applied to small-scale development projects. In the classroom the process described in this paper was developed and used over consecutive six semesters in four different courses. Specifically the process was introduced in the graduate course Spatial and Temporal Design where it has been used three times. It was then used for the undergraduate course Level Design and Game Architecture where again it has been used three times and most recently added to undergraduate courses Foundations of Game Design where it has been used once. Additionally the developer Christopher Totten has used this process to develop the level design paradigm for his studio's current project and by the Google and ArenaNet developer Lincolin Hughes to investigate the foundations of social design.

These applications of the process were undertaken with several goals. First, to use the process of deriving a pattern to teach a specific design principle. Second, to allow a cohort of developers to create a shared design vocabulary that both serves their specific needs and is understood by all participating developers. Third, to help developers create an individual conceptual design framework that fits their learning style and development needs rather than providing rote learning of existing frameworks such as Mechanics Dynamics Aesthetics (MDA) [17] or Design Lenses. [26]

In order to achieve these goals, it was necessary to create and codify the process of pattern creation. In general, the process of creating a pattern has been omitted in the literature, even within Alexander's work. Takashi Iba discussed his methods in some detail, but his process was designed for a large group able to dedicate significant resources to the development of a language. [18] For active use in a classroom and in small-scale development, a more concise scalable approach would be needed. 


\section{Proposal for individual patterns and languages}

The project undertaken to meet these goals was to develop a repeatable process for creating patterns that could be executed by designers of any skill level, and which would produce patterns that could be clearly understood and used by any designer familiar with the process. Further, this process of language creation allows designers to link the patterns they created, as well as existing patterns, together to form case-specific pattern languages that can produce reliable solutions to complex design problems. Finally, the process allows individual developers to organize their personal design knowledge as best fits their needs.

\section{Materials and Methods}

To satisfy these needs, I created a pattern template that could be used to make sure that all patterns would contain all of the same ontological structure. The pattern template created was based on the original ideas of Alexander as laid out in his seminal work A Pattern Language: Towns, Buildings, Construction. I incorporated ideas from the patterns of Björk \& Holopainen as well as Adams and Dormans where they were in line with my understanding of Alexander's intent. Additionally I added elements that I felt were needed to support learning through the process of pattern creation as well as the use of the created patterns in practical design.

In order to ensure a uniform application of this template, as well as facilitate the use of patterns and pattern languages recorded using the template, I created a web based tool for entering and accessing patterns. This tool uses a relational database to store pattern data. The creation of this database schema implied an ontological structure which will be discussed later in the Proposed Ontology for Pattern Language for Game Design Patterns section. Additionally the creation of a public facing repository for recording patterns necessitated the consideration of issues of privacy and access for patterns recorded using this tool. Aspects of the template that relate to these concerns are presented in the second optional part of the template as they are not intrinsic to patterns, but are integral to this practical implementation of a pattern library.

Using this template, a series of exercises were created and implemented in a classroom setting. The resulting patterns were reviewed in terms of how well they served the goals derived from the proposal for individual patterns and languages:

- Pattern reflects or is not in conflict with validated design principles

- Pattern can be clearly understood and used by any designer

- Pattern describes its links with other existing patterns

- Pattern addresses the specific problem of the designer creating it

As stated in the section An alternative application of patterns, these exercises were iterated on over the course of six semesters of courses. The resulting pattern derivation process and language creation process are described below.

Pattern creation and application was incorporated into three courses at Northeastern University. Each course covered a long standing aspect of the game design curriculum in their respective programs. Some of these aspects are course-specific, such as the architectural and level design understanding in the Spatial and Temporal Game Design course and the Architecture and Level Design course. Other aspects are general and span all 
courses in the curriculum, for example the ability to analyse existing design and understand its purpose or to look at specific design techniques and consider their effects across diverse audiences. At the time that this method was introduced to these courses none of the students had encountered the method in a previous course. Going forward the effect of students encountering the method in a foundational course and then revisiting it in advanced and graduate courses will be evaluated. The specifics of how the below method was incorporated into these courses is detailed in the Discussion of pattern exercises as part of curriculum section.

\section{The iterative process resulting from common problems}

The creation of the process described in this paper has been very iterative. Patterns were first introduced as a part of course pedagogy before the format of exercises had been established. After each use of patterns in course work, the structure of exercises and the specifics of the template used to record patterns was refined. Additionally the process of linking patterns together into a language was initially performed in a very manual way where all students reviewed each pattern in the initially small library and looked for connections. As the library grew the techniques of using keywords, pattern seeds, and recommended exercises were added. Much of the functionality of the pattern library website was developed in support of this process.

\section{Revised pattern definition/template}

In order for the patterns created by this process to meet the stated goals, each pattern must contain all of a set of properties and may contain additional optional properties. The required parts of a pattern are:

- Pattern Title

- Design Problem

- Pattern Description

- Pattern Confidence

- Author(s)

- Keywords (Keywords, Categories, Properties)

- $\quad$ Example Games (Name, Example Description)

The optional parts of a pattern are:

- Pattern State

- Pattern Seed

- Groups(s)

- Pattern Image

- Pattern Image Description

- $\quad$ Related Patterns (Name, Description, Confidence)

- $\quad$ Suggested Exercises

Definitions of required properties are presented here. 
Pattern Title: The name of the pattern. Alexander advised that the name of a pattern should be both descriptive and evocative. In practice creating a good title can be challenging as it is also important not to create a title that requires a niche cultural context.

Design Problem: The general design problem that the pattern serves as a solution to. When setting out the template and displaying patterns it is important to privilege this property as it is common for developers to spot a pattern and document it without considering its design problem as seen in Game Design Patterns [6] and early student patterns in this project.

Pattern Description: This is the heart of the pattern, a description of the actual pattern. The description has a length between one paragraph and one page. It is important to give enough context to make the pattern easy to implement and to call out drawbacks or concerns about the pattern. In general beginning with a sentence like "In order to [achieve some design effect], a designer may [take some design action, use some mechanic, etc.] because [explanation of how the pattern produces the desired effect]."

Pattern Confidence: This is a numerical reflection of the author's confidence in the accuracy and effectiveness of the pattern. This number is generated using a confidence rubric that will be presented below. The addition of a formal rubric for confidence is an enhancement to the idea of confidence as introduced by Alexander in A Pattern Language. [2]

Author(s): All of the designers who contributed to the creation of the problem.

Keywords: A listing of all of the keywords that relate to the pattern. This is critical in linking patterns together when they do not have a more structured connection listed in the property of the related pattern. Keywords are also important as they can be used at the time of pattern creation to search the library for other patterns that may need to be listed in the property of the related pattern. In the development of this process, a curated list of keywords was used. This list was expanded each semester, but limiting the list helps to encourage a more limited set of keywords that avoids synonyms and redundancies such as using 'Third Person' and '3rd Person.'

Example Games: Because, as Alexander defined it, the nature of a pattern is to be the "core solution to that problem, [stated] in such a way that you can use the solution a million times over, without ever doing it the same way twice" [2] it is necessary to include examples of the pattern's use in a diverse set of games. The process of derivation which is discussed below, generates this list of games and capturing it here allows other designers to take the generalized pattern which can be abstract and see it applied in concrete ways.

Definitions of the optional properties are presented here.

Pattern Image: An iconic image to represent each pattern. This image can help to convey the essence of the pattern and to serve as a mnemonic anchor for remembering it. Designers with animation talent have had good luck creating animations to demonstrate the application of their patterns. 
Pattern Image Description: A short written description of the image is both clarifying and important for accessibility in patterns intended for public use.

Related Patterns (Name, Description, Confidence): This property is optional only because there may not be any related patterns, especially in new libraries with a small number of preexisting patterns. In a mature library most if not all new patterns will be connected to one or more other patterns as parents, children, additive, subtractive, or alternate patterns. These structured connections are the primary axioms that structure patterns into a language. All of these relationships are recorded using the following format:

Related Pattern Name (Confidence: $n$ ): Description of how the relationship functions.

Different pattern projects have used different terminology for these connections, or used slight variations on these concepts so I will describe each relationship axiom below.

Parent Patterns: A pattern or several patterns that are needed by this pattern for it to function well. Typically this follows the form; Bjork and Halopanien use the term 'Is Instantiated By.' This is accurate and descriptive, but I have chosen less formal and possibly less accurate language to appeal to a broader audience.

Child Patterns: Patterns that are suggested by this pattern or require it to function well. Again Björk and Holopainen use the term 'Instantiates.'

Additive Patterns: A pattern is considered to be additive when its presence makes another pattern more effective, but when each can function without the other.

Subtractive Patterns: A subtractive pattern makes another pattern less effective. Björk and Holopainen group additive and subtractive patterns together under a 'Is Modulated By' axiom, which does make clear that you might want to include a subtractive pattern as part of balancing gameplay.

Alternate Patterns: Two patterns are alternates of each other when they both solve the same design problem, but in different - and possibly mutually exclusive - ways.

One axiom that is not represented in the current ontology is the idea of an arbitrary incompatible pattern, one pattern that causes another not to function rather than just modulating its effectiveness. The pattern derivation process that I presented below simply does not uncover this relationship. However, the use of patterns derived from this process may well expose this type of axiom and it may be added to the ontology in the future.

Pattern State: This state reflects the level of completion and privacy of the pattern. This is idiosyncratic to a shared pattern library with many users. In order to foster the use of a library in as many ways as possible, this project allows patterns to be in a draft state where only the authors can view the pattern, in a review state where any groups that the authors are members of can view and comment on it, or in a published state where the pattern is visible by anyone. There may be additional states related to the maintenance 
and administration of a library such as marking a pattern as deprecated or merged with another duplicate pattern.

Pattern Seed: This indicates the specific exercise and design element that was used to derive the pattern. Capturing this information is necessary for the evaluation of the effectiveness of each exercise in producing the intended types of patterns.

Group(s): This property represents an arbitrary collection of patterns. For instance, all the patterns created by a particular team of designers, or by students belonging to a specific course section, or associated with a university or studio. This property is at the time of this writing unique to this project. This information is only important in largescale or public pattern libraries. However, in these cases, it allows both privacy and the ability to easily represent smaller pattern languages within a larger library of patterns. Patterns may be included in multiple groups allowing them to be shared by use-specific languages.

Suggested Exercises: Often during the process of creating a pattern designers note multiple other possible patterns. It is not always practical to document all of these patterns immediately. This property is used to record these nascent patterns so that developers can pursue them at a later time.

\section{Proposed Ontology for Pattern Language for Game Design Patterns}

It is valuable to codify the above properties and axioms using a formal ontology language. The OWL 2 ontology [34] was chosen for this purpose. While only a simple definitional ontology has so far been created the long-term goal is to apply a robust ontological structure to all patterns created in a programmatic way and to instrument the pattern library allowing existing reasoner software to facilitate the selection of pattern languages that address complex sets of design problems. This application of the proposed ontology will be the subject of ongoing research.

Because the Pattern Library website uses a MySQL RDB to store all data related to the patterns entered there, an ontology can be programmatically applied by creating declarations for all of the classes and object properties in the ontology and then using the existing PHP/JavaScript code to produce individuals for all of the patterns in the library, or in a specific language stored in the library.

A simplified representation of the ontology is presented here in Figures $x, x$, and $x$ The current set of classes and object properties being used are presented in formal OWL2 Manchester syntax in the supplementary materials section.

\section{Classes}

\begin{tabular}{|l|l|l|l|}
\hline example_game & game_platform & keyword & pattern_suggested_exercise \\
exercise & game_publisher & pattern & related_pattern \\
game & game_release & pattern_exercise & user \\
game_available_link & game_type & pattern_related_pattern & EquivalentTo: author \\
game_developer & group & pattern_seed & author \\
game_info_link & group_type & pattern_states & EquivalentTo: user \\
\hline
\end{tabular}

Figure 1. Ontology classes for representing a pattern 


\section{Object Properties}

\begin{tabular}{|l|l|}
\hline Object Property - Domain - Range & Object Property - Domain - Range \\
\hline $\begin{array}{l}\text { hasAuthor - pattern - user } \\
\text { hasExampleGame - pattern - example_game }\end{array}$ & $\begin{array}{l}\text { hasGroupType - group - group_type } \\
\text { hasKeyword - game, pattern - keyword } \\
\text { hasExercis - pattern - pattern_exercise }\end{array}$ \\
hasGame - example_game - game & hasPatternSeed - pattern - pattern_seed \\
hasGameAvailableLink - game - game_available_link & hasRelatedPattern - pattern - related_pattern \\
hasGameDeveloper - game - game_developer & hasRelease - game - game_release \\
hasGameInfoLink - game - game_info_link & hasSuggestedExercise - pattern - pattern_suggested_exercise \\
hasGamePlarform - game - game_platform & hasUser - pattern - user \\
hasGamePublisher - game - : game_publisher & owl:topObjectProperty - related_pattern - pattern \\
hasGameType - game - game_type & relatesTo - related_pattern - pattern \\
hasGroup - pattern, user - group & \\
\hline
\end{tabular}

Figure 2. Ontology object properties for representing a pattern

\section{Data Properties}

\begin{tabular}{|l|l|}
\hline Data Property - Domain & Data Property - Domain \\
\hline $\begin{array}{l}\text { game_available_link_notes - game_available_link } \\
\text { game_available_link_source - game } \\
\text { game_available_link_url - game }\end{array}$ & $\begin{array}{l}\text { pattern_exercise_name - pattern_exercise } \\
\text { pattern_exercise_page - pattern_exercise } \\
\text { game_description - game }\end{array}$ \\
pattern_image - pattern \\
game_developer_name - game_developer & pattern_image_description - pattern \\
game_developer_notes - game_developer & pattern_name - pattern \\
game_image - game & pattern_seed_description - pattern \\
game_info_link_notes - game_info_link & pattern_seed_name - pattern \\
game_info_link_source - game_info_link & pattern_state_name - pattern \\
game_info_link_url - game & pattern_suggested_execise_description \\
game_name - game & pattern_suggested_exercise \\
game_plarform_name - game_platform & related_pattern_confidence - related_pattern \\
game_platfrom_notes - game_platform & related_pattern_description - related_pattern \\
game_publisher_name - game_publisher & related_pattern_type - related_pattern \\
game_publisher_notes - game_publisher & video_gameplay - game \\
game_release_date - game & video_trailer - game \\
game_release_name - game_release & group_type_description - group_type \\
game_release_notes - game_release & group_type_name - group_type \\
game_release_type - game & pattern_confidence - pattern \\
game_type_name - game_type & pattern_created_date - pattern \\
game_type_notes - game & pattern_description - pattern \\
group_auto_join - group & pattern_design_problem - pattern \\
group_name - group & pattern_example_game_description - example_game \\
\end{tabular}

Figure 3. Ontology data properties for representing a pattern

\section{Pattern Exercises}

As discussed in the introduction a robust pattern library -while useful - is only one part of this project. The goal is to make the creation of a pattern language a repeatable process and to enable any designer to engage in the creation of patterns that fit the set of design problems they face.

Over the course of six semesters, and in three distinct courses, a variety of exercises were created to guide designers - students in this case -in creating patterns. At the time of writing twenty six exercises have been created and tested. The majority of these pattern exercises are presented in the book Pattern Language for Game Design. [3] The first of these will be discussed below. Students have used these exercises to create upwards of 400 
patterns. In the past year, patterns have been recorded in the Pattern Language For Game Design website. [4]

\section{Basic Pattern Exercise}

Step 1: Name a design element.

Step 2: Name ten games that use that element-the more different ways the games use it, the better.

Step 3: Describe how each of those games uses the element you chose. Try not to look for a pattern yet. Focus on accurately describing the way each game uses the element you identified.

Step 4: What design problems do the games use the element to solve? Some games may use the element for one purpose, while others use it for another. Many games use the elements in more than one way. Describe the problems solved by your element for each of the ten games listed in step 2.

Step 5: Look at steps 3 and 4. Are there patterns in the ways the games use the element, and how do those relate to the problems they solve?

Step 6: Pick one of those patterns and describe it using the pattern template.

Step 7: You may repeat step 6 for each pattern you observed.

Figure 4. Statement for Basic Pattern Exercise as presented to students

The Basic Pattern Exercise as presented in Figure 4 presents a seven-step process for creating a pattern. It is important to note that this process does not begin with a design problem, though other exercises do. In this case the designer is asked first to choose a design element to investigate through the exercise. For the purpose of this exercise a design element is defined as an aspect of design that can be either intrinsic or extrinsic to the game as an artifact, and either formal or functional. For example, a platform in a side scrolling game would be an intrinsic formal element, whereas a game controller would be a formal extrinsic element. The player-controlled character's ability to jump would be an intrinsic functional element.

After selecting a design element, the designer is instructed to name ten games that make use of that element. There are several considerations in this step of the process. The exercise instructs the designer to choose games that use the design element as differently as possible. This is the result of observing that patterns generated by choosing diverse games are broader, more generalizable, or higher level than those created by looking at games within a genre. This is desirable, particularly when the designer is just learning the process and when creating a new pattern language. As the designer's skills develop or if the pattern language is already robust then more focused specific patterns might be desired.

A second concern is the number of games examined. A large sample size is good but examining more than ten games makes the process prohibitive for a single designer. If an exercise is being completed by a group, then more than ten games should be examined. It may also be the case that a designer has not played ten games that use the design element they are focusing on. In this case it is necessary to research additional games. Playing them is preferable but reading reviews and watching gameplay is also useful. 
Once a set of games has been selected the designer is instructed to describe how the games use the element. The designer is also cautioned against beginning the process of searching for a pattern. This was a common mistake on the part of students during the development stages of this learning method. After naming one or two games the students would decide on a pattern and select the rest of the games in a manner that confirmed their prematurely formed pattern. The resulting patterns were usually still valid but had a much narrower scope and often overlooked examples that would have clarified the pattern, revealed circumstances where it was less effective, or resulted in a higher-level pattern. It is not possible to eliminate this type of confirmation bias entirely, but it can be reduced by focusing on clear documentation of the use of the technique at this point.

Having produced a set of descriptions of design element use, the designer next considers what design problem each element's use solves. This process is subjective and is dependent on the insight of the designer. Though it is important to note this element of subjectivity, it does not invalidate the produced pattern; rather it results in patterns that reflect the insights of the particular designer. The patterns produced by new designers tend to describe design principles that are already familiar to experienced designers. These simple patterns can be an important part of a larger pattern language, even when it is being shared with experienced designers. $M$ importantly they allow the newer designer to deeply understand the design principle they have expressed. This has been a primary benefit to using these techniques in the classroom.

Often several implementations of a design element address the same problem, or a single implementation addresses several disparate design problems. Thus, in the following step the designer notes all of the possible patterns that relate to their chosen element.

Finally, the designer selects one of the possible patterns and records it using the pattern template, selecting and describing appropriate example games. This last step may be repeated for each of the noted patterns.

\section{Connecting Patterns into a Language}

As described in the pattern template section of this paper, patterns as conceived of by Alexander do not exist independently, they exist as part of a highly connected language. The process of identifying these connections provides significant benefit for both the utility of the language and for the learning process of the designers producing the language. In the ontology used here these connections take the form of keywords and related patterns properties.

When introducing a new pattern into an established language it is relatively easy to make these connections using the process described below. However, in the early development of a language it may be the case that few or no related patterns yet exist. In this case it is necessary to continue developing patterns as needed and reexamining the library with each new addition to see if new connections should also be created.

Over the course of this project the following process has been successful for language formation.

1. A shared set of keywords is applied to all patterns that have been created. It is important that this set of keywords is curated and limited to accurately code patterns that share concepts. 
2. All patterns that share a keyword are noted and reviewed by their authors. If either author believes that there is a relationship between two patterns its type is discussed and agreed upon by both authors. Relationships are usually reciprocal and thus each author needs to add the relationship to their pattern.

3. For patterns that have suggested exercises, those exercises are completed, and the resulting patterns are linked if appropriate.

4. If a pattern does not have any related patterns at this point, then specific exercises targeted at creating higher or lower-level patterns are completed using the same seed as the orphan pattern. Resulting patterns are linked as indicated.

Pattern collections resulting from this process have been dense enough to constitute a useful language in of the six semester long iterations of this process. However, these languages have consisted of 80 to 117 patterns and are not considered to be functionally complete, or even sufficient to fully serve as a basis for pattern driven game-development. In the past four iterations of this process all patterns were entered into the Pattern Library Website and the resulting languages were interconnected. The growing size of the Pattern Library Website will require a process of regular trimming to remove low quality patterns and combine patterns that are functionally identical.

\section{Discussion of pattern exercises as part of curriculum}

The results of the integration of pattern exercises into the curriculum have been discussed above as they apply to the refinement of the pattern and the language creation process. From the perspective of students, the generation of a repeatable process and a mature library are secondary to the role that the creation process plays in the curriculum.

In each course that incorporates patterns, students are introduced to the process of completing pattern exercises early in the course. They are then presented with different exercises depending on the content of the course. For instance, students in the introductory 'Foundations of Game Design' course complete exercises to create patterns based on formal and functional elements of design. Students in the graduate level 'Spatial and Temporal Design' course complete more complex exercises based on player circulation patterns or on boss encounter design.

In all courses students immediately use the patterns they create in small design projects that show how they would apply the pattern to solve its stated design problem. The result of one such exercise is included in the Supplementary Materials section of this paper.

Each course progresses to the use of patterns to create a language and includes a final project in which students describe a complex design problem and then identify a small set of patterns that they believe will help them solve that problem. They then produce a midsized project over the last weeks of the course. This project is then analyzed by other students in the class to both see how well the patterns were implemented and whether they contributed to the solution of the stated design problem.

\section{Example of a student-created Pattern Language}

The following example shows a sample design problem and the pattern language used to address it, The full design document for the project is available in supplemental Materials. 


\section{Design Problem}

"Can fast and reaction based movement be made easier for newer players?"

\section{Architecture Boundaries}

\section{Patterns}

Clear immersive boundaries make it easy to show where players can and cannot use their mechanics, and function as an obstacle the player must navigate to avoid while moving at fast speeds. In our case, we have hills and trees surrounding the player, which both adds to the theme of enchanted forest as well as provides a clear, logical boundary to the track.

\section{You Mute, You Lose}

A design pattern that incorporates using specific sound effects/music that allows the player to be notified of incoming actions as well as their result. Utilizes sound effects to also allow players to be familiar with the pacing of that level.

\section{Teach Me How to Fight}

When players begin a game it is important to show them basic mechanics by introducing smaller less meaningful targets to practice on. In our case, our enemies are static and introduced in small packs to the player.

\section{Let's Take Things Slow}

A mechanics based pattern, this directly relates to our design problem as it describes how to slow down fast-paced action in games. Specifically, with difficult maneuvers or when players are first starting out, incorporating slowed-time movements or actions is helpful to grant players more time if their reaction times aren't quick enough yet. Incorporating affordances like a physical timer that allows players to hit a button within the time range, or bullet time when attempting fast-paced or high-precision movements will be helpful to aid newer players.

\section{I'm Doing it as Hard as I Can}

Gradually increasing the difficulty for a new player will help give them the time to become more adept at basic mechanics so that they may use them at higher levels later on. We used this for our turns (making the turns walled, then unwalled, then tighter turns), introducing our jumping on ramps mechanic and drifting/boosting mechanic in safer spaces earlier on in the track, and in the slow introduction to enemies in the track.

\section{This is About to Hurt}

Players can better prepare for incoming obstacles or battles when they are made aware of an upcoming engagement. When new players are learning a game, icons and modular assets can help them be made aware of what type of engagement is coming and how to properly react to that. One example in our game is our enemies' particles that are emitted and visible from a distance, potentially before the enemies themselves.

\section{Let's do it Again}

When beginning to tutorialize a player, giving them the ability to repeat without the fear of failure allows them to strengthen their understanding of what they are learning. Checkpoints also allow the player to repeat difficult portions of a level. Combined with an infinite live/restart system, players are able to go at their own pace before feeling accomplished.

\section{Chaos is the greatest form of Fun!}

Random elements in a competitive setting are usually not welcome, but their inclusion allows for less skilled players to be on a more equal playing ground, keeping their enjoyment alive.

\section{Sitting Through the Previews}

In a game that may have a lot of action going on that could be overwhelming for new players, 
having some kind of safe space or pause in gameplay that allows them to just observe the physical space they'll be dealing with can be helpful for them to plan their strategy ahead of time and know what to expect. In our case, we actually created a quick 6 or 7 second cutscene that had multiple cameras panning over different areas of the track before the player began.

Figure 5. Sample student created pattern language

Each of the above patterns was developed during the course of the semester. Some of the patterns were developed by this team specifically to address this design problem, but others were created by other teams earlier in the semester. All created patterns were linked using keywords and pattern relationships allowing this team to discover a set of patterns that met their needs.

\section{Example of pattern use in an industry context}

Discussions with industry professionals have yielded the consistent opinion that fully pattern driven development is impractical for a studio just beginning to use patterns. Thus the adoption of such a development methodology would be at best a gradual process, and possibly not feasible given real world production constraints. However, more limited use of pattern exercises and language to solve a specific design problem has proven successful in at least one case.

Developer Christopher Totten of Pie For Breakfast Studios has used these techniques to establish a level design paradigm for his successfully Kickstarted game Little Nemo and the Nightmare Fiends. His team was having trouble finding a shared frame of reference for their level design style. Totten conducted several pattern exercises and the team produced several patterns that they were able to use to define, understand, and agree upon the paradigm they would use for level design within the game. One of the primary patterns is shown and linked and described below. 


\section{Central Travel Axis}

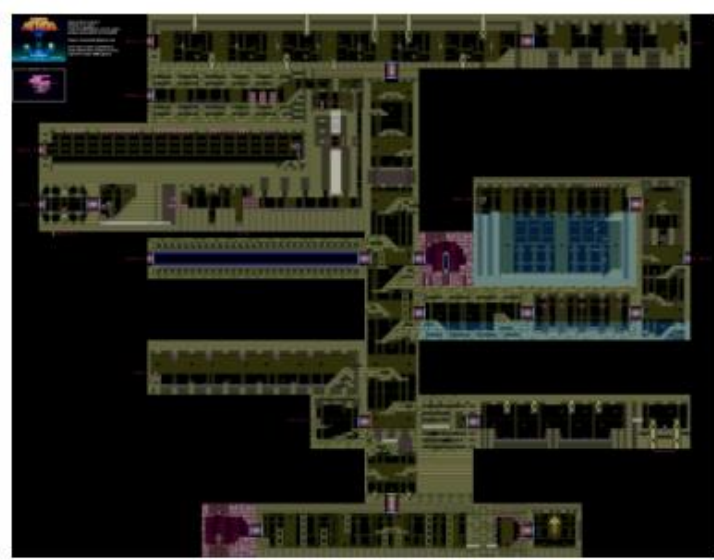

\begin{tabular}{|c|c|c|}
\hline Author(s): & $\therefore$ totter & \\
\hline \multicolumn{3}{|l|}{ Groups(s): } \\
\hline Pattern & \multirow{2}{*}{\multicolumn{2}{|c|}{4}} \\
\hline Confidence: & & \\
\hline Created On: & \multicolumn{2}{|c|}{ May 24 th $2021,3: 13 p m$} \\
\hline Edited On: & \multicolumn{2}{|c|}{ June 15 th $2021,9: 58 \mathrm{am}$} \\
\hline Seed: & \multicolumn{2}{|l|}{-} \\
\hline \multirow[t]{4}{*}{ Keywords: } & Exploration & Level Design \\
\hline & \multicolumn{2}{|l|}{ Metroidvania } \\
\hline & Adventure & Boundaries \\
\hline & Good Shape & \\
\hline
\end{tabular}

Design Problem:

Pattern

Description:

Example Games:

How to lower the burden of repeat visits to a level (backtracking.) How to signify player progress when they revisit places in a game world.

In games, especially those with large worlds that allow open-ended exploration, zones are organized around one or several primary spaces that allow for quick and easy passage, in contrast with other parts of the zone which may test the player's abilities. These spaces may be horizontally or vertically oriented. A common trope (perhaps a pattern to be added onto this one) is to have these spaces blocked or inaccessible during a player's first visit to a zone, but open it up as the player gains abilities or unlocks gates/changes to the environment.

\section{Hollow Knight}

Hollow Knight is a 2D "Metroidvania" style game. Zones have vertical or horizontal versions of these pattern. They are blocked off initially via a gate or boss fight, but the player opens them up after taking the "long way around" through challenging areas that bring players to a point where they may open up the room where the level may be traversed easily.

\section{Castlevania: Aria of Sorrow}

Areas of Dracula's castle feature large open areas with multiple exits that the player travels through multiple times as they explore. These areas allow for quick traversal. Initially the player may only reach a few exits due to their limited abilities, but as they grow they gain more freedom to move through these areas in any way they wish.

\section{Shantae: The Seven Sirens}

Dungeons have vertical shafts that allow quick travel between areas, but these are not opened up until the player gains the dungeon's special item. Before the special item is found, travel requires traversal through dangerous areas.

\section{Super Metroid}

Most zones have vertical passages that serve as a "spine" of the area, from which tunnels and other explorable areas branch from. Exits to the side tunnels are usually open from the beginning, but still rendered inaccessible until the player has gained specific powers.

Figure 6. Example designer created pattern

\section{Results}

This project focused on the application of pattern theory in pedagogy and design practice rather than being structured as a formal study. Formal experimental results can not be provided at this stage. However, a significant amount of data was generated in the form of completed design patterns and graded student projects. This growing data set can be formally assessed in future research. Additionally initial observations made during this project can be offered.

\section{Peer review and course grading}


The results of the pattern exercises that were developed over the course of this project were assessed in several ways. The most direct was through grading by the instructor. Each pattern was considered in the context of existing design theory. In most cases students did not enter into the exercises with formal knowledge of this design theory. The consistency with which students articulated known design theory and with which the sophistication of that theory exceeded their previous understanding suggests that the exercise process was effective in helping students synthesize their experiential design knowledge, and research, into formal theory.

A second form of assessment was used in which students assessed each other's patterns and design projects. These assessments were then reviewed by the instructor. In assessing pattern exercises, the ability of the reviewing student to understand the theory being articulated by the students producing patterns was considered. In project assessment, the ability of the assessing students to identify the ways that patterns were implemented in the projects was considered. In most cases the reviewing students' ability to perform both of these tasks and correctly identify and clearly articulate design techniques in their peer's work was higher than in previous courses that had not used patterns as part of the curriculum This suggests that the patterns produced were an effective method of communicating design ideas between developers and in helping students integrate design ideas introduced in course texts.

Specifically for the course that produced the above example students were assigned projects from other teams and asked complete the following exercises:

1. Look at the design document of your assigned group.

a. Compare it to what you saw in the demonstration.

b. In your readings and assignments document, list each pattern the group used.

c. Describe how well you think it was implemented and whether it had its intended effect.

2. List as many techniques from Game Design and Architecture as you can find in their design. For each technique:

a. Is it part of a pattern, which one?

b. Does the technique help solve the group's design problem? How?

c. What techniques do NOT help solve the design problem?

d. Do the techniques that do not relate to the design problem support techniques that do? If not, could they be removed?

This exercise produced two to three pages of analysis from each student. A few representative excerpts are included below. These are indicative of the level of understanding acquired by the students through the process of pattern derivation and use.

In response to exercise 1 :

\section{Teach Me How to Fight (analyzed by Colin Yang)}

I think the track/level itself serves as the "smaller target" for players to practice the game's mechanics on. The level includes all of the mechanics listed in the design and doesn't punish the player much if they fail. As such, I think the pattern serves its purpose well. 


\section{Let's do it Again (analyzed by Colin Yang)}

The design includes checkpoints that respawn the player at the beginning of the section for the mechanic that they failed. The track is also loopy, so that if the player fails, they land right back on the track, also making it easier to get right back into it. I think this pattern is implemented well.

I'm Doing It As Hard As I Can (analyzed by Michael Iantosca)

This pattern is concerned with gradually increasing difficulty in order to compensate for a player's increase in skill level. This pattern actually applies to group 4's final level in a number of ways. Firstly, like I mentioned earlier, the more complex bits of the track come later on in a circuit, and scaling difficulty is inherent to any racing game, as the better the player becomes the faster they will be able to get around the circuit.

Students accurately identified the ways that each pattern had been applied to the project games. When there were flaws in the implementation of the patterns, analyzing students identified them in a nuanced way and often suggested changes in implementation that would improve the effect of the pattern in the game.

In response to exercise 2:

\section{Montessori Building Blocks (analyzed by Harrison Sims)}

Pattern: This technique is connected to Teach Me How to Fight and Let's Do it Again because the technique is used to teach and then test, but over the course of the game. As the game goes on, I would expect the designers to teach the player new techniques that can be used to complete levels.

Analysis: This technique does help new players with fast and reaction gameplay because it is about teaching the player how to play the game basically, and the teaching will make the player better at the desired gameplay.

\section{Access as a First Level Reward (analyzed by Harrison Sims)}

Pattern: Architecture Boundaries and Let's Do it Again both apply to this technique, but for different reasons. Analysis: Architecture Boundaries because areas are "locked" until the player passes the current area using the new skill, like the jump or the power up loop. Let's Do it Again because in sections like the jump or the power up loop the player has multiple attempts to complete the task, and the reward is moving to the next section of the map. I think [the technique] does help with the design problem because it makes sure the player is able to accomplish the skills necessary for the fast and reaction based movement of the later levels.

\section{Negative space (analyzed by Michael Iantosca)}

Pattern: This technique relates to the pattern It's Not All About Looks

Analysis: It helps define the boundaries of the play space. It helps to solve the design problem by creating a clear, continuous circuit for new player to traverse. The negative space created by the lack of high mountains and trees in the track immediately cue the player in on where they are supposed to be going and clearly define where the boundaries are. 
Local symmetry (analyzed by Michael Iantosca)

Pattern: I'm Doing It As Hard As I Can.

Analysis: The track makes use of symmetries by repeating challenges from earlier in the track later on with increased challenge and complexity. In this way, it relates to the pattern I'm Doing It As Hard As I Can. It helps solve the design problem by allowing new players to try the challenges of the circuit multiple times in order to get better at them, even within a single run.

In the responses to this second exercise students show that they can connect the many design techniques that they have learned about in the class outside of the pattern process. These techniques are presented through the text for the class and through lecture and discussion. The ability to relate these techniques to the patterns that they have derived during the course grounds their evolving personal design frameworks with established design theory.

\section{General observations across student and developer projects}

A number of broad trends are visible across student developer patterns and projects. The initial patterns produced often state design principles that the developer is already familiar with. The type of pattern produced is easily influenced by the context in which the developer is working, whether it be a particular design project, or the material being covered by a course. Although game designers need to be aware of this effect, it is not necessarily detrimental since it allows the developer to focus on the problem they are currently facing. Early attempts at completing pattern exercises are time consuming. Developers tend to gain speed with early repetition, but eventually slow in their process again as they learn to use the exercises to look for patterns relating to techniques that they don't already understand well. When using patterns in projects groups of developers are able to make use of the patterns that they have become familiar with to create a shared vocabulary and communicate complex design concepts efficiently.

\section{Mapping the Teaching and Learning Functions to the pattern creation process}

As part of the process of assessing the efficacy of using patterns as a pedagogical framework it is useful to apply a widely understood set of pedagogical techniques such as the learning and teaching functions proposed by Shuell and Moran. [27] The consideration of each of these twenty-two functions in respect to the specific steps in the creation and application of patterns as discussed here yields a potential mapping of their functions to this process. This mapping seems to be direct and robust enough to merit further formal analysis and suggests that this application of pattern theory could provide the basis for a viable pedagogy for the teaching of game design. The proposed mapping is shown in full below.

\section{Preparation}

- Prior knowledge activation: Designers are asked to choose seeds for the patterns they develop based on their prior knowledge or in the case of students' concepts that have just been introduced through course texts.

- Motivation: Completion of the pattern exercise process is required by the developer's team mates as well as for the completion of game projects, both of these provide external motivation for developer persistence and contribution.

- Expectations: Exercises are introduced with full examples and designers completing them identify uses of the pattern as part of the process naturally identifying the utility of the process they are completing. 
- Attention: In order to identify patterns and clarify their use in example games designers must isolate the formal and functional techniques that those games contain which relate to their exercise.

\section{Knowledge Manipulation}

- Encoding: Developers create their own patterns deciding how to express the ideas they have observed. They choose the pattern title and image to help solidify the concept the pattern captures.

- Comparison: During the first stages of the pattern process designers compare the use of their seed technique across existing games. Later in the process they identify the best applications of the pattern that they have articulated as it is applied in existing games and choose the most diverse applications as examples to include in their pattern.

- Repetition: Within a specific iteration of the process of pattern discovery designers analyze ten or more games looking for uses of their seed technique. The larger process of language creation involves repeating the pattern creation process many times and reviewing the created patterns looking for connections.

- Interpreting: Designers must examine existing games which contain the techniques they are investigating, understand the use of those techniques and then articulate the shared aspects of their purpose and implementation in the form of a pattern. Later they must begin with a pattern and design a game that implements the pattern to achieve the previously stated purpose.

- Exemplifying: When completing an exercise developers must provide examples of the use of the pattern. These examples usually differ from the games that were analyzed as the source for the pattern. Additionally, designers are encouraged to find the most diverse set of examples possible to illustrate the scope of their pattern.

\section{Higher Order Relationships}

- Combination, integration, synthesis: Individual patterns are created by observing and combining the purpose and implementation of techniques across games. Pattern languages are created by articulating the relationships between patterns in terms of subject, purpose and function.

- Classifying: Each pattern must be assigned a set of keywords to place it within the context of existing design theory. Three levels of keywords are provided: keywords which identify the patterns' subject matter, categories which place it in an area of design, and properties which indicate its purpose.

- Summarizing: The description of the pattern is a summary of the analysis that the designer has undertaken to derive the pattern.

- Analyzing: Patterns are created through the analysis of a set of existing games, these must be decomposed and understood in terms of the seed technique of the pattern exercise.

\section{Learner Regulation}

- Feedback: As part of the language creation process patterns are peer reviewed and revised to best form the connections necessary for the language.

- Evaluation: On project completion projects are peer reviewed to analyse the efficacy of their implementation of the patterns. 
- Monitoring: During the use of patterns in design projects the implementing designers provide feedback to the designers that developed each pattern.

- Planning: The use of patterns in practical design projects is intrinsically a planning process wherein the designers use patterns to structure their design prior to implementation.

\section{Productive Actions}

- Hypothesis generation: The process of pattern formation consists of analyzing data and forming a hypothesis.

- Inferring: Designers take existing design knowledge, examine existing examples of its use and infer the patterns that it forms.

- Explaining: Creating the textual artifact of a pattern using the provided template allows designers to articulate and explain the theory they have constructed. Patterns are then further used to explain the more complex composite concepts that form a complete game design.

- Applying: Using patterns as the basis of design in practical game projects allows designers to apply the concepts that they have articulated and validate their efficacy.

- Producing and constructing: From simple scene implementations using a signal pattern to complex full game designs the practical execution of design into game provides designers with the opportunity to demonstrate their learning in functional game artifacts.

\section{Discussion}

\section{A rigorous review process and future metrics}

As the basic process for creating patterns and applying them to design projects has solidified it has become more feasible to design a rigorous set of metrics for measuring the accuracy and efficacy of patterns.

This process must include:

a stable rubric for assessing patterns in terms of existing design theory;

a persistent way to record the use of patterns in student projects;

standardized assessment of playtest data for games using patterns;

assessment of games developed by equivalent students without the use of patterns;

a larger sample of industry developers producing and using patterns.

The rapid, iterative and largely informal process used to develop this pedagogy has been remarkably productive, but in order to understand and validate the extent of its usefulness more formal study is clearly required. The empirical study design used by Florez et al. in their Teaching Software Engineering Topics Through Pedagogical Game paper is applicable here with minor modifications. This study model will be applied in the Games@Northeastern: Studio Project discussed below.

\section{Limited project size}

One of the major limitations in the observed use of patterns to produce game projects in the classroom is the limited scope of the projects themselves. In each course seven oneweek projects are completed followed by a single four week project. The shorter projects 
produce a playable scene, and the four-week project produces a longer scene with more polish. These short projects often clearly show the function of a pattern, but they do not show its efficacy in the context of a complete game.

\section{The benefits and limitations of student developers}

The use of this process with students has produced higher quality analysis and clearer articulation of the design techniques used to produce projects. This effect may or may not be reflected in developers of greater experience. Every derived pattern provides new insight for students, and it is possible that experienced designers may derive less benefit from the process. To date only three experienced designers have completed a significant number of pattern exercises and related their experience. All three have found the process useful and derived patterns that addressed their needs, but this number is far too low to make any generalization.

\section{Games@Northeastern: Studio Project}

During the Summer of 2021 a new project has begun at Northeastern University to allow students to undertake large scale development using patterns to guide design. In this project up to fifty students will participate each semester for a year to produce a single complete game. This development process will be monitored from its inception to more rigorously evaluate the effect of patterns on its design. The outcomes of that project will be presented when they become available.

The design of this project is intended to leverage the use of patterns both to maximize student learning in the process and to allow all of the students in the studio to contribute to the design and have visibility into the high level and detailed design. The basic structure of the design process is as follows. Over the Summer of 2021 students will all contribute design ideas and collectively select the most interesting subset. Selected ideas will be decomposed into their atomic mechanics and design techniques. Pattern exercises will be completed with these seeds. The resulting patterns will be connected into a pattern language. In five teams, students will examine the resulting language and select a subset of patterns that are densely connected to form the basis for a final design. One design will be selected, and all students will participate in the development of that game over the Fall and Spring semesters.

\section{Conclusions}

The idea of a pattern language was introduced by Alexander almost 45 years ago in a different field of study. Today the idea of patterns is commonly understood in game design, however the potential utility of a pattern language in game design education and practice has not been realized. Having identified omission of design problems, presumption of functional completeness at the level of pattern languages, narrow topical focus, and lack of a concise, repeatable method for pattern production as shortcoming in existing implementations of patterns in game design a more robust method for applying patterns to game design was created.

This new method addressed the above weaknesses through introducing a new pattern template, creating a defined process for connecting patterns into a language and assessing the scope of that language, creating a rubric for assessing pattern confidence and interconnectivity confidence, and generating an introductory set of pattern-building exercises. This method was iteratively applied in the classroom and by several industry designers allowing for its refinement and assessment. 
This use of the pattern creation and application methods discussed in this paper allowed the students and developers involved to

- $\quad$ access the learning opportunities offered by the process of creating patterns

- utilize the communication clarity created through a shared understanding of a pattern

- $\quad$ practice thoughtful design through connecting practical design problems with the patterns that solve them

On a small scale the process has been successful in use with students and in limited use by developers in industry. However, more extensive rigorous study of the process and its results is needed and has begun.

\section{Supplementary Materials:}

\section{Basic Pattern Exercise Example}

Step 1: Name a design element.

Jumping

Step 2: Name ten games that use that element-the more different ways the games use it, the better.

Donkey Kong/Jumpman, Q*bert, Super Mario Bros. (Braid as subversion?), Mirror's Edge, Gravity Rush/VVVVVV, Alice/Super Mario World/Crackdown, Guild Wars 2, Tomb Raider (reboot), Prince of Persia (second reboot), Poptropica, Super Meat Boy, Street Fighter/Soulcalibur/Devil May Cry, Doom/Quake/Splosion Man, Tribes, Assassin's Creed, Canabalt, Sonic, Trials HD

Step 3: Describe how each of those games uses the element you chose. Try not to look for a pattern yet. Focus on accurately describing the way each game uses the element you identified.

Jumping is such a fundamental design element that I tried to go back and select games that first used the mechanic in historically significant ways, as well as listing the most modern and innovative uses of the mechanic. Initially, only six examples of significance jumped into my head, so I did a small amount of research to see if there were general opinions on important uses of jumping. The willingness to pause and research a question like this is essential to the pattern development process since we all have played a limited number of games.

- Donkey Kong/Jumpman, Geometry Dash - Jumping is used to avoid enemies and traverse the 2D space.

- $\quad Q^{*}$ bert-A reflex-based puzzle game made in the wake of Pac-Man, it uses jumping as its only movement mechanic.

- Super Mario Bros. - Jumping is used to avoid enemies, traverse 2D/3D space, and as a way to attack enemies.

- $\quad$ Mirror's Edge-This game uses first-person jumping as pure traversal.

- Gravity Rush/VVVVVV - Jumping combines with control of physics.

- $\quad$ Alice/Super Mario World/Crackdown - Jumping with a glide. Also, in-air control?

- Guild Wars 2-Jumping for exploration and as a puzzle. There's little need for it in world traversal, and none in combat.

- Tomb Raider (reboot)-Your ability to jump in this game is superhuman despite the more realistic tone of the game.

- Prince of Persia (second reboot) - This game features assisted jumping where another character helps you jump farther than you can alone. The game is single-player so that may be just a double jump. 
- Doom/Quake/Splosion Man/Tribes - Jumping for world traversal. Jumping assisted by the physics of unrelated systems (Rocket Jumping, Ski Jumping, Bunny Hopping).

- Street Fighter/Soulcalibur/Devil May Cry - Jumping for world traversal, jumping as a combat move.

- $\quad$ Poptropica, Super Meat Boy - Jumping for world traversal with very unrealistic physics.

- Trials HD-Jumping in unrealistic environments with very realistic physics.

- Assassin's Creed-Jumping “on rails” for world traversal, jumping to escape enemies.

Step 4: What design problems do the games use the element to solve? Some games may use the element for one purpose while others use it for another. Many games use the elements in more than one way. Describe the problems solved by your element for each of the ten games listed in step 2.

- Navigation through the world space (all games listed) - All the games I chose used jumping as part of world traversal. At a base level, jumping gives you more movement options as a player.

- Creating a sense of autonomy in the player (all games listed)-Jumping increases a character's mobility in the game world, and that can give you a feeling of greater agency as you play. There's some subtlety in how this works, though. In some games, characters can jump in a way that more closely mirrors the real world, which can make you relate to the character more. In other games, jumping lets the character move through the world in ways you never could in real life, which can still feel empowering because you're controlling the character.

- Creating a sense of danger for the player (Super Mario Bros., Mirror's Edge, Gravity Rush/VVVVVV, Super Meat Boy, Tomb Raider, Prince of Persia, Trials HD) - Jumping can cause death in all of these games. The ratio of how dangerous jumping is to how much it lets you traverse the world directly relates to the amount of power versus fear that you feel when you play. In a game like Super Mario Bros., you may die from jumping incorrectly, but mostly it increases your ability to navigate the world. In a game like Geometry Dash, jumping allows you to progress through the world, but it's also the main thing that causes you to die when you do it incorrectly.

- Adding variety to the ways the player can interact with the world - All games listed except $\mathrm{Q}^{*}$ bert and Geometry Dash, in which jumping is the only way you move through the world. But this is particularly true in Guild Wars 2, where jumping isn't a primary world traversal tool and is mostly used in optional jumping exploration puzzles.

- Enabling player mastery through creating complicated, intricate systems that require player skill growth-All games listed. It seems like this connection is stronger the more central jumping is as a mechanic, and the more complicated and subtle the jump mechanics are.

- Enabling player mastery of game systems by creating opportunities for the player to subvert them (Doom/Quake, Tribes) - This is interesting since, in the case of these games, the mechanics were not meant to allow player subversion. Rocket jumping and ski jumping were, on some level, bugs that players found and used to enhance gameplay. The developers, recognizing the value of the bugs, intentionally incorporated them into future games.

- Character building through giving the character abilities the player lacks (Mirror's Edge, Gravity Rush, Alice, Tomb Raider, Poptropica, Assassin's Creed)-Superhuman jumping abilities help make the characters seem superhuman, but not inhuman. Improving a character's most basic movement abilities lets you relate to the character-I can jump, but not like that! - in a way a completely inhuman ability wouldn't.

- Maintaining immersion in the game world by making character abilities and movement match your understanding of how the real world works - Interestingly none of the example games above use jumping in this way, but other games do (e.g., Silent Hill 2, Flashback).

- Enhancing combat by enhancing aggressive player actions (Street Fighter/Soulcalibur, Super Mario Bros., Devil May Cry) - The jumping itself may not be aggressive, but it amplifies the character's aggressive action. A jumping punch to the head is just more impactful than a standing punch to the head.

- Step 5: Look at steps 3 and 4. Are there patterns in the ways the games use the element, and how do those relate to the problems they solve?

- Yes.

- More complex mechanics provide more opportunities for player skill. This taps into basic player needs like autonomy and mastery. 
- When power has a cost, it's frightening to use. This would generate a pattern of dangerous jumping.

- Two great things that go great together, such as jumping and punching. This might be a very specific pattern about those two mechanics, or it might generalize to "movement and attack" or even to pairs of mechanics that create a player experience together that is more than either can produce alone.

- She's just like me! vs. I want to be her when I grow up! This would create a pattern about maintaining immersion by creating realistic character abilities vs. character building through superhuman abilities.

Step 5: Pick one of those patterns and describe it using the pattern template. When power has a cost, it's frightening to use.

Step 6: You may repeat step 6 for each pattern you observed.

For this example, I will only document one pattern. In the next section, I'll show the completed writeup for the pattern I chose. Describing the other three patterns using the Pattern Template is an excellent way to practice before completing the full exercise on your own.

\section{Research:}

Creating even simple patterns like this can take a huge amount of both generalized game knowledge and knowledge of specific games. It's easy to feel like the more you know about games and the more games you have played, the better patterns you will see. That's true to a degree, but you shouldn't feel like there's no point in trying to create a pattern because you don't know enough yet. If you're a new designer, the patterns you see may be basic, but they're also probably fundamental. Experienced designers may fail to identify relevant patterns because they're too obvious. That said, when you're constructing a pattern, do try to find at least ten examples. If you can't think of that many games off the top of your head, do some research. The following sources are from the research I did when I was creating this pattern. If you look at the citations, you'll see that I'm referencing a scholarly article, an article on a popular gaming website, a Reddit post, and a fan-made games FAQ website. It's vital to evaluate your research sources and understand how much rigor or opinion there is in what you read. But it's also essential to look beyond academic analysis and consider both media perception and player experience. I do not list the background research for each pattern in the book, but I commonly read a dozen or more sources as I am investigating a possible pattern.

"The Rise of the Jump" (Butler 2014)

"What Was the First Game with a Double Jump and Why Was It Implemented?" (reddit 2016)

"You Say Jump, I Say How High? Operationalising the Game Feel of Jumping" (Fasterholdt, Pichlmair, and Holmgård 2016)

“What Game Do You Think Has Perfect Jumping Mechanics?” (GameFAQs 2018)

\section{Notes on this pattern}

Pattern Name

As stated in the Pattern Template, the pattern name should be an "easy to remember and evocative name." There's a fine line between easy to remember and an inside joke or reference to a fleeting meme. Titles should be evocative, but they shouldn't be a reference that only you or your close friends will understand. Think about who will be reading your pattern and make sure that the title, image, and example games are understandable to the developers who will need to use the pattern.

Description and Example Games

You may notice that this pattern doesn't have anything specifically to do with jumping. That's not an accident. Many students will see jumping as the pattern itself rather than just a mechanic. Designers tend to want to take the games they've listed in steps 2 and 3 of the exercise and list those as their examples. But it's essential to look at the actual pattern you have generated and find examples of its use that are not examples of the functional element from step 1. If you see many natural examples of wildly different implementations of the pattern you've identified, it's a good indication that you've found a viable pattern. If you find examples that only relate to your starting functional element, then make sure that your pattern description reflects that narrow focus.

\section{Pattern}



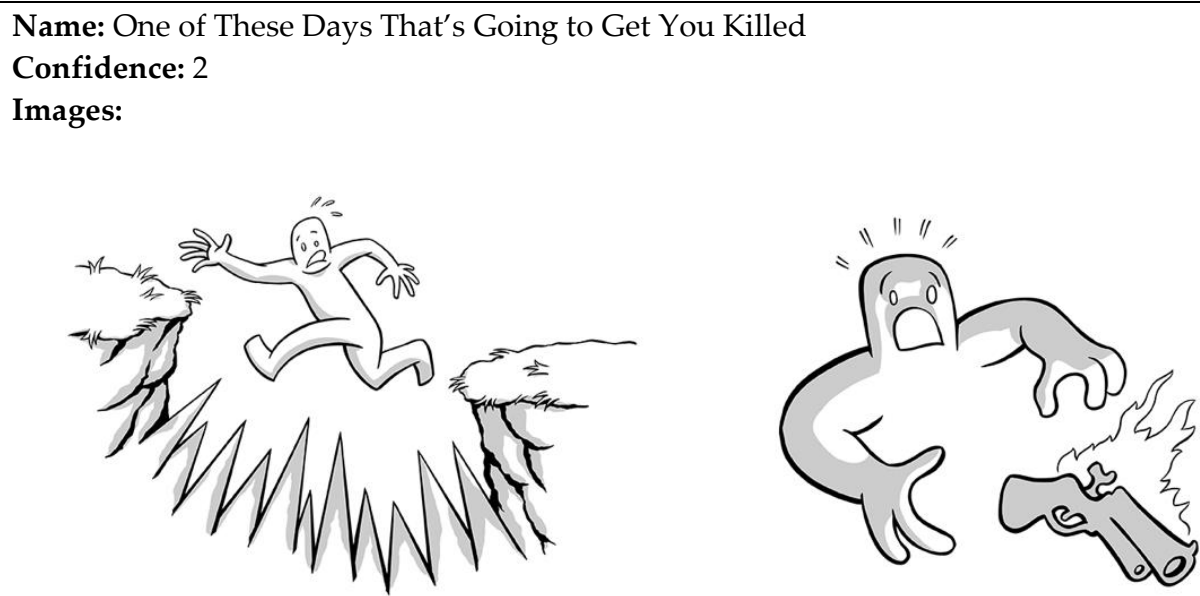

Jumping over a dangerous pit and suffering from a weapon overheating are both examples of this pattern in action.

\section{Author: Chris Barney}

Design problem: How do you maintain game balance and create tension when giving the player greater power in their interactions with the game world?

Description: To maintain balance and create tension when designing character abilities, a designer may introduce consequences resulting from using those abilities. The result may be something natural, like falling into a pit of lava you try to jump over, or it may be something mechanical, like weapon heat build-up or a stamina meter.

\section{Games that use this pattern and how:}

- Super Mario Bros. - The ability to jump, which increases the character's ability to move through the world and defeat enemies, also puts him in danger. Failing to jump over dangerous obstacles can result in Mario's death. Similarly, failing to jump over an enemy results in the enemy killing Mario.

- Sekiro: Shadows Die Twice-Stealth-killing enemies is the easiest way to defeat them, but failing to execute a stealth kill alerts the enemy and nearby enemies and suddenly puts you in a dangerous situation.

- Anthem-Firing weapons increases their heat. Failing to manage that resource, to selflimit the damage you are doing, can result in not being able to fire your gun when you most need it.

- Zelda: Breath of the Wild - Link can climb almost anything, but he has a stamina meter, so if he tries to climb something too high he will fall. He can jump off things and glide, but if he runs out of stamina, he falls to his death.

Seed: Exercise 1: Basic Pattern Exercise-Jumping

\section{Related patterns:}

\section{Parent patterns:}

Just Look At What You've Become (Confidence: 2)-As you apply the pattern One of These Days That's Going to Get You Killed you are introducing consequences for player actions and character advancement. Use this pattern to ensure that character progression is a meaningful transformation.

I'm Doing It As Hard As I Can (Confidence: 3)-The escalating difficulty introduced by this pattern creates the mechanical need for the character progression systems produced by One of These Days That's Going to Get You Killed.

\section{Suggested Exercises}

Use Exercise 5: Functional Patterns to generate a pattern based on the functional element of mechanical character progression. 
And Now I Guess We Are Doing This (Confidence: 3) - When you use One of These Days That's Going to Get You Killed to balance increases in character abilities, you create situations where the character is in peril. You can use these situations to force the player to adjust their playstyle using this pattern.

The Risk of Knowing You (Confidence: 2) - When you use One of These Days That's Going to Get You Killed to balance increases in character abilities, you both place the character in danger and create a sense of risk for the player. Use this pattern to help you use those two effects to create a stronger bond between player and character.

\title{
Suggested Exercises
}

Use Exercise 4: Formal Patterns to generate a pattern based on environmental hazards.

Use Exercise 5: Functional Patterns to generate a pattern based on character stamina.

Use Exercise 5: Functional Patterns to generate a pattern based on limited ammunition.

Keywords: Character Progression, Mechanics, Balance

\section{One of these days that's going to get you killed.}

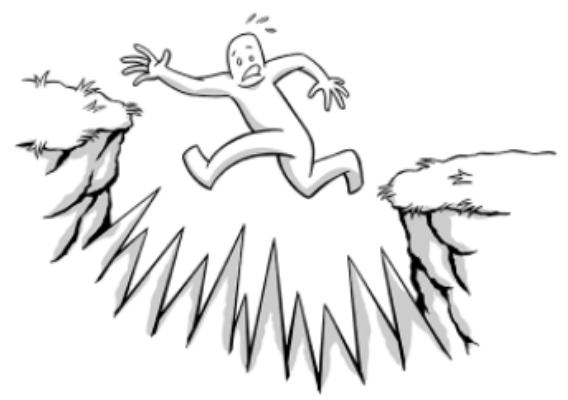

\author{
Author(s): \\ Groups(s): \\ Pattern \\ Confidence: \\ Created On: \\ Edited On: \\ Seed: \\ Keywords:
}

$\therefore$ Chris Barney

Pattern Language for Game Design 2

June 13th 2020, 11:14am

November 16 th 2020, 9:10am

Exercise 1: Basic Patterns Exercise - Jumping

Balance Character Progression

\section{Mechanics}

Jumping over a dangerous pit and suffering from a weapon overheating are both examples of this pattern in action.

Design Problem: How do you maintain game balance and create tension when giving the player greater power in their interactions with the game world?

Pattern To maintain balance and create tension when designing character abilities, a designer may introduce consequences

Description: resulting from using those abilities. The result may be something natural, like falling into a pit of lava you try to jump over or it may be something mechanical, like weapon heat build-up, or a stamina meter.

Example Games:

\begin{tabular}{|c|c|}
\hline Super Mario Bros. & (i) \\
\hline \multirow{2}{*}{$\begin{array}{l}\text { The ability to jump, which increases the character's ability to movt } \\
\text { in danger. Failing to jump over dangerous obstacles can result in } \mathrm{N} \\
\text { results in the enemy killing Mario. }\end{array}$} & Game Description - Super Mario Bros. \\
\hline & \multirow{2}{*}{$\begin{array}{l}\text { The ability to jump, which increases the character's ability to move through the world } \\
\text { and defeat enemies, also puts him in danger. Failing to jump over dangerous } \\
\text { obstacles can result in Mario's death. Similarly, failing to jump over an enemy results } \\
\text { in the enemy killing Mario. - View Game Details }\end{array}$} \\
\hline Sekiro: Shadows Die Twice & \\
\hline \multicolumn{2}{|c|}{ 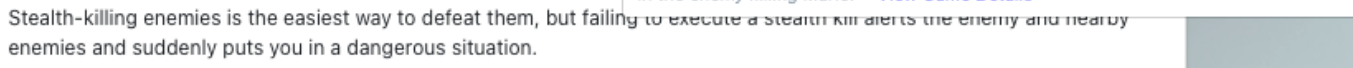 } \\
\hline Anthem & (i) \\
\hline
\end{tabular}

Figure 8. Pattern as it appears on the patternlanguageforgamedesign.com website. Web interface allows navigation through keywords and display of supporting information such as details about example games. 
The full proposed OWL2 ontology is presented in Manchester syntax as that is the most human readable and compact. Following the ontology definition a sapling of individuals is provided. All object properties are SubPropertyOf: owl:topObjectProperty and all data properties are SubPropertyOf: owl:topDataProperty. These have been omitted from the below ontology for brevity. Ontology header information has been omitted for brevity. Text has been reformatted and ordered for readability.

Ontology: http://www.semanticweb.org/christopherbarney/ontologies/2021/5/pattern-ontology

\section{Classes}

\begin{tabular}{|l|l|l|}
\hline Class: example_game & Class: game_release & Class: pattern_seed \\
Class: exercise & Class: game_type & Class: pattern_states \\
Class: game & Class: group & Class: \\
Class: game_available_link & Class: group_type & pattern_suggested_exercise \\
Class: game_developer & Class: keyword & Class: related_pattern \\
Class: game_info_link & Class: pattern & Class: user \\
Class: game_platform & Class: pattern_exercise & EquivalentTo: author \\
Class: game_publisher & Class: & Class: author \\
& pattern_related_pattern & EquivalentTo: user \\
\hline
\end{tabular}

Figure 9. Classes from the pattern language ontology

\section{Object Properties}

\begin{tabular}{|c|c|c|}
\hline $\begin{array}{l}\text { ObjectProperty: hasAuthor } \\
\text { Domain: pattern } \\
\text { Range: user }\end{array}$ & $\begin{array}{l}\text { ObjectProperty: hasGamePlarform } \\
\text { Domain: game } \\
\text { Range: game_platform }\end{array}$ & $\begin{array}{l}\text { ObjectProperty: hasPatternState } \\
\text { Domain: pattern } \\
\text { Range: pattern_states }\end{array}$ \\
\hline $\begin{array}{l}\text { ObjectProperty: hasExampleGame } \\
\text { Domain: pattern } \\
\text { Range: example_game }\end{array}$ & $\begin{array}{l}\text { ObjectProperty: hasGamePublisher } \\
\text { Domain: game } \\
\text { Range: game_publisher }\end{array}$ & $\begin{array}{l}\text { ObjectProperty: hasRelatedPattern } \\
\text { Domain: pattern } \\
\text { Range: related_pattern }\end{array}$ \\
\hline $\begin{array}{l}\text { ObjectProperty: hasExercis } \\
\text { Domain: pattern } \\
\text { Range: pattern_exercise }\end{array}$ & $\begin{array}{l}\text { ObjectProperty: hasGameType } \\
\text { Domain: game } \\
\text { Range: game_type }\end{array}$ & $\begin{array}{l}\text { ObjectProperty: hasRelease } \\
\text { Domain: game } \\
\text { Range: game_release }\end{array}$ \\
\hline $\begin{array}{l}\text { ObjectProperty: hasGame } \\
\text { Domain: example_game } \\
\text { Range: game }\end{array}$ & $\begin{array}{l}\text { ObjectProperty: hasGroup } \\
\text { Domain: pattern, user } \\
\text { Range: group }\end{array}$ & $\begin{array}{l}\text { ObjectProperty: hasSuggestedExercise } \\
\text { Domain: pattern } \\
\text { Range: pattern_suggested_exercise }\end{array}$ \\
\hline $\begin{array}{l}\text { ObjectProperty: hasGameAvailableLink } \\
\text { Domain: game } \\
\text { Range: game_available_link }\end{array}$ & $\begin{array}{l}\text { ObjectProperty: hasGroupType } \\
\text { Domain: group } \\
\text { Range: group_type }\end{array}$ & $\begin{array}{l}\text { ObjectProperty: hasUser } \\
\text { Domain: pattern } \\
\text { Range: user }\end{array}$ \\
\hline $\begin{array}{l}\text { ObjectProperty: hasGameDeveloper } \\
\text { Domain: game } \\
\text { Range: game_developer }\end{array}$ & $\begin{array}{l}\text { ObjectProperty: hasKeyword } \\
\text { Domain: game, pattern } \\
\text { Range: keyword }\end{array}$ & $\begin{array}{l}\text { ObjectProperty: owl:topObjectProperty } \\
\text { Domain: related_pattern } \\
\text { Range: pattern }\end{array}$ \\
\hline $\begin{array}{l}\text { ObjectProperty: hasGameInfoLink } \\
\text { Domain: game } \\
\text { Range: game_info_link }\end{array}$ & $\begin{array}{l}\text { ObjectProperty: hasPatternSeed } \\
\text { Domain: pattern } \\
\text { Range: pattern_seed }\end{array}$ & $\begin{array}{l}\text { ObjectProperty: relatesTo } \\
\text { Domain: related_pattern } \\
\text { Range: pattern }\end{array}$ \\
\hline
\end{tabular}

Figure 10. Object Properties from the pattern language ontology

\section{Data Properties}

\begin{tabular}{|l|l|l|}
\hline $\begin{array}{l}\text { DataProperty: } \\
\text { game_available_link_notes }\end{array}$ & $\begin{array}{l}\text { DataProperty: game_release_date } \\
\text { Domain: game }\end{array}$ & $\begin{array}{c}\text { DataProperty: pattern_exercise_name } \\
\text { Domain: pattern_exercise }\end{array}$ \\
\hline
\end{tabular}




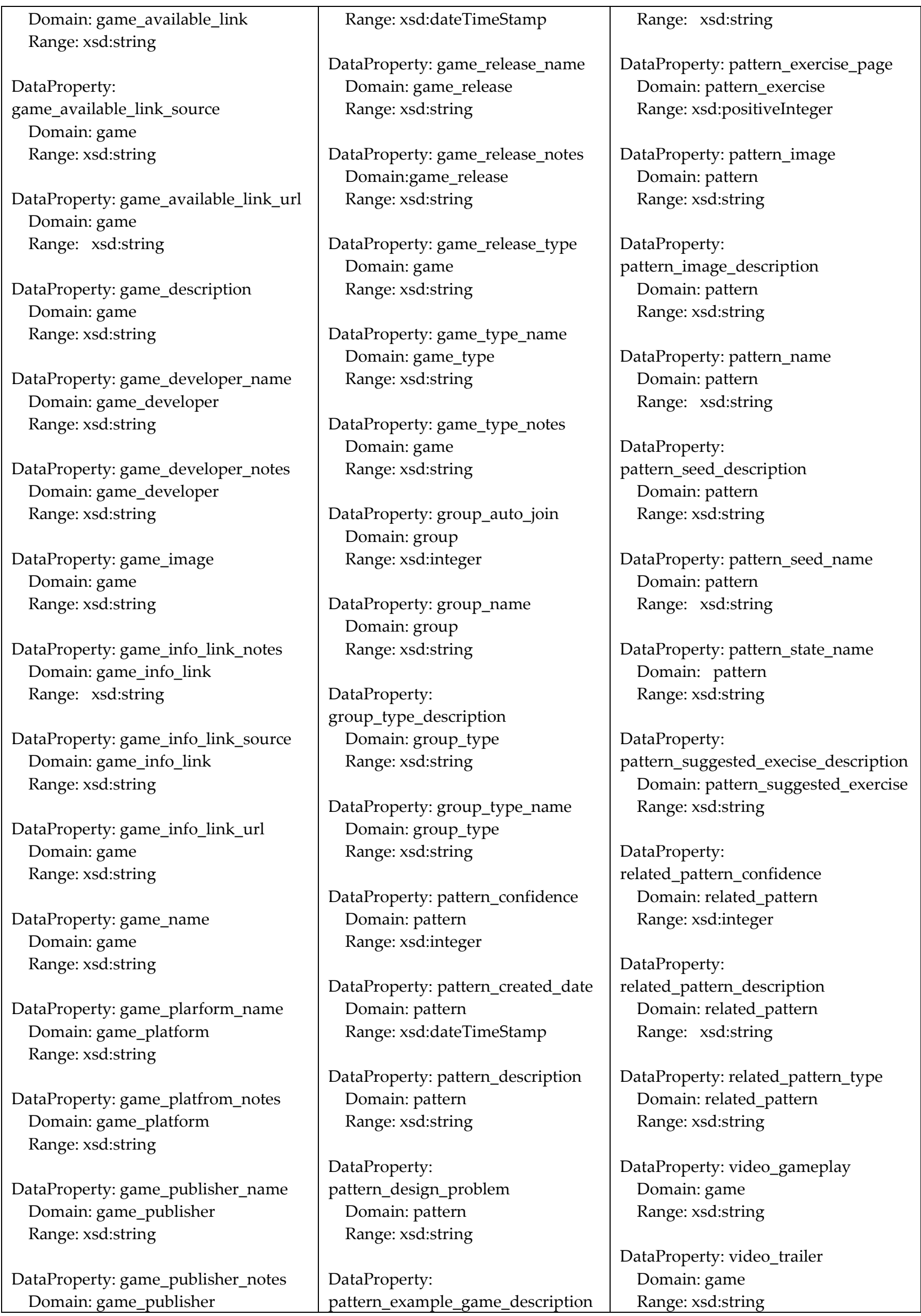




\begin{tabular}{|l|l|l|}
\hline Range: xsd:string & Domain: example_game & \\
& Range: xsd:string & \\
& DataProperty: & \\
& pattern_exercise_description & \\
& Domain: pattern_exercise & \\
& Range: xsd:string & \\
\hline
\end{tabular}

Figure 11. Data Properties from the pattern language ontology

\section{Example Individuals Representing a Single Pattern and its Associations}

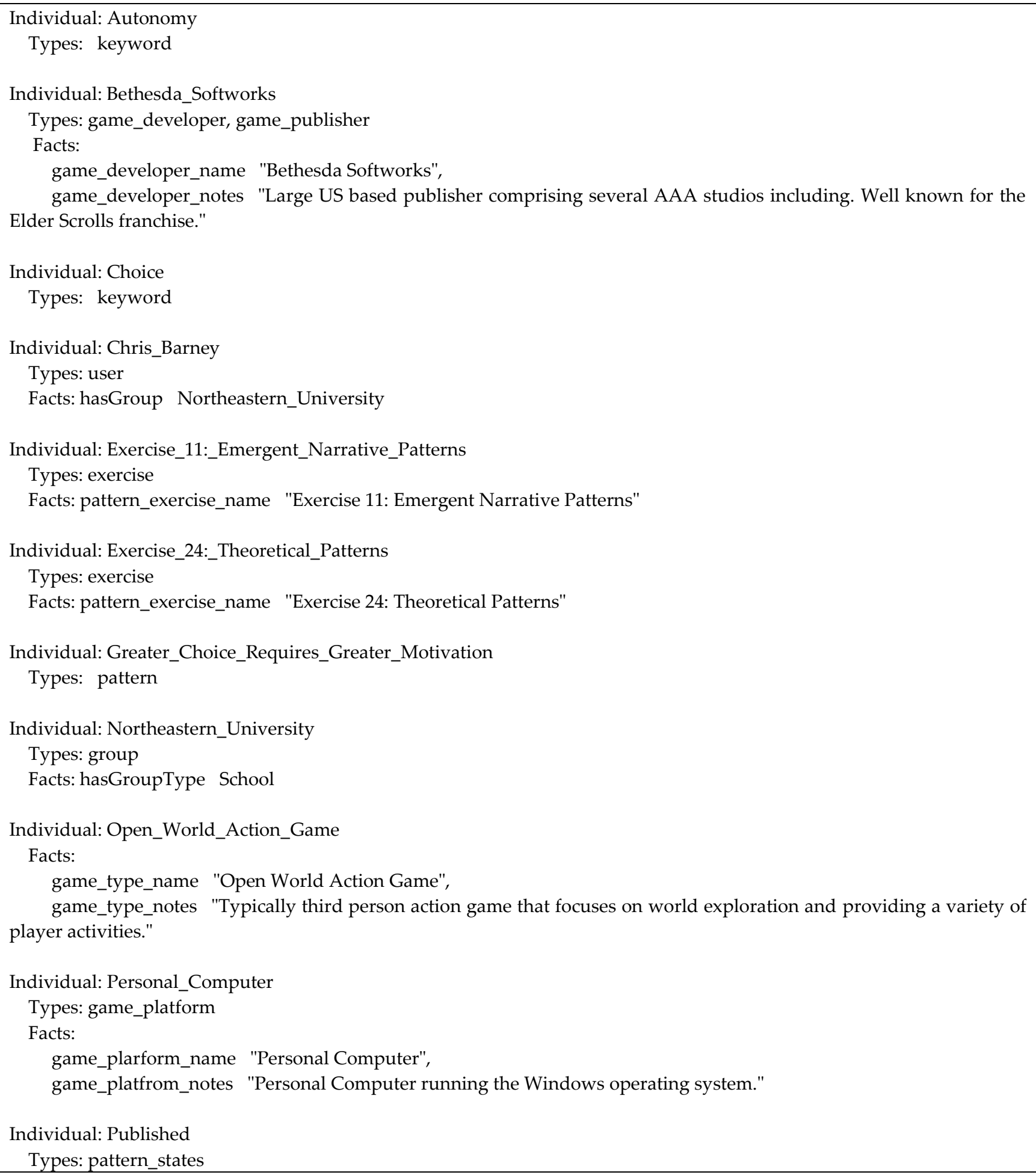




\footnotetext{
Facts:

pattern_state_name "Published"
}

Individual: School

Types: group_type

Facts:

group_type_description "Educational institution, college, or university teaching game design and producing design patterns.",

group_type_name "School"

Individual: Skyrim_Available_Link_1

Types: game_available_link

Facts:

game_available_link_notes "Steam download for the Special Edition release of the game.",

game_available_link_source "Steam",

$\mathrm{n} / "$

game_available_link_url "https://store.steampowered.com/app/489830/The_Elder_Scrolls_V_Skyrim_Special_Editio

Individual: Skyrim_Info_Link_1

Types: game_info_link

Facts:

game_info_link_notes "'",

game_info_link_source "Official Website",

game_info_link_url "https://elderscrolls.bethesda.net/en/skyrim"

Individual: Skyrim_Primary_Release

Types: game_release

Facts:

game_release_date "01/01/2011",

game_release_name "Primary",

game_release_notes "Initial release on Windows PC",

game_release_type "Primary"

Individual: The_Elder_Scrolls_V:_Skyrim

Types: game

Facts:

hasGameAvailableLink Skyrim_Available_Link_1,

hasGameDeveloper Bethesda_Softworks,

hasGameInfoLink Skyrim_Info_Link_1,

hasGamePlarform Personal_Computer,

hasGamePublisher Bethesda_Softworks,

hasGameType Open_World_Action_Game,

hasRelease Skyrim_Primary_Release,

game_description "Skyrim is the fifth instalment in the Elder Scrolls universe. It is an Action RPG in either first or

third person, set in an open-world. Sandbox style nonlinear play is exhaustive. Thousands of quests and npc driven mini plots are available, as well as the main storyline quests. The primary character is fully customizable and upgrades in all skills and equipment make for a very wide variety of play experiences. Only Single-player mode is available.",

game_image "[Skyrim Image URL]",

game_name "The Elder Scrolls V: Skyrim",

video_gameplay "JSRtYpNRoN0",

video_trailer "JSRtYpNRoN0"

Individual: Three_Pillars_Relationship_1

Types: related_pattern

Facts:

relatesTo Greater_Choice_Requires_Greater_Motivation, related_pattern_confidence 2, 
related_pattern_description "When you have applied The Three Pillars of Meaning to situations where there are emergent narrative and player choices, then those choices will be meaningful. The more significant you make choices, the more of them your game will be able to support.",

related_pattern_type "Child"

Individual: Three_Pillars_Seed

Types: pattern_seed

Facts:

pattern_seed_description "What makes emergent events narratively meaningful?",

pattern_seed_name "Seed for Three Pillars of Meaning in Emergent Narrative"

Individual: Three_Pillars_Skyrim_Example

Types: example_game

Facts:

hasGame The_Elder_Scrolls_V:_Skyrim,

pattern_example_game_description "The degree to which the three pillars are present for emergent narrative elements in this game varies. At worst, the events are isolated and unrelated to the player or the world: a group of bandits in a cave with no associated NPCs or consequences for "ridding the countryside" of them. At best, all are present: killing an NPC in town results in the guards becoming hostile, the character attracting the attention of the assassins' guild, and the inability to wear holy armor due to your evil actions."

Individual: Three_Pillars_Suggested_Exercise_1

Types: pattern_suggested_exercise

Facts:

hasExercise Exercise_24:_Theoretical_Patterns,

pattern_suggested_execise_description "Use Exercise 24: Theoretical Patterns to generate a parent pattern based on the theory that The Three Pillars of Meaning in Emergent Narrative is generalizable to narrative in general."

Individual: Three_Pillars_of_Meaning_in_Emergant_Narrative

Types: pattern

Facts:

hasAuthor Chris_Barney,

hasExampleGame Three_Pillars_Skyrim_Example,

hasExercise Exercise_11:_Emergent_Narrative_Patterns,

hasGroup Northeastern_University,

hasKeyword Autonomy,

hasKeyword Choice,

hasPatternSeed Three_Pillars_Seed,

hasPatternState Published,

hasRelatedPattern Three_Pillars_Relationship_1,

hasSuggestedExercise Three_Pillars_Suggested_Exercise_1,

pattern_confidence 2,

pattern_created_date "12/22/2020",

pattern_description "To allow players to construct meaningful emergent narratives, developers should provide players with context, motivation, and consequence for their actions in the game. There are many child patterns that contribute to this pattern; two are listed and 12 other possibilities are provided in the suggested exercise, and there may be more. But each one contributes to either the context of, motivation for, or consequences of an event that could be part of an emergent narrative.",

pattern_design_problem "Given the reality of limited resources, when creating design elements that encourage emergent narratives, designers need to maximize the narrative potential of every element. How can designers know if a given game element will contribute to meaningful emergent narratives?",

pattern_image "pattern_image.jpg",

pattern_name "Three Pillars of Meaning in Emergent Narrative"

Figure 12. Example Individuals from the pattern language ontology 
Funding: This research received no external funding.

\section{Data Availability Statement:}

\section{Acknowledgments:}

The analysis of the methods discussed in this paper would not have been possible without the dedicated course work of the students in the GAME and GSND programs at Northeastern University. In particular this paper cites example patterns and analysis from the following students as representative of the larger body of analysed work: Simon Astor, Izzy Conner, Liam Cristello, Meredith Goujon, Magen Hocker, Becca Malcolm, Michael Iantosca, Leylanah Mitchell, Danny Son, Jack Speake, Harrison Sims, Liam Sean Thornton, and Colin Yang. Additionally the patternwork of the industry designers Christopher Totten and Link Hughes is appreciated.

Author Contributions: Conceptualization, methodology, software, validation, writing-original draft preparation, Christopher A. Barney; writing - review and editing, Pedro Neves. All authors have read and agreed to the published version of the manuscript.

Conflicts of Interest: The author declares no conflict of interest. The founding sponsors had no role in the design of the study; in the collection, analyses, or interpretation of data; in the writing of the manuscript, and in the decision to publish the results.

\section{References}

1. Adams, Ernest, and Joris Dormans. Game Mechanics: Advanced Game Design. Berkeley, CA: New Riders Games, 2012.

2. Alexander, Christopher (1977). A Pattern Language: Towns, Buildings, Construction. Oxford University Press, USA. p. 1216. ISBN 978-0-19-501919-3.

3. Barney, Christopher A. Pattern Language For Game Design. S.l.: CRC Press, 2020.

4. $\quad$ Barney, Christopher A. "Pattern Library." Pattern Language for Game Design. December 22, 2020. Accessed July 16, 2021. https://patternlanguageforgamedesign.com/.

5. Björk, Staffan. "Gameplay Design Patterns." Gameplay Design Patterns Collection. August 8, 2019. Accessed April $20,2020$. http://GameplayDesignPatterns.org.

6. Björk Staffan, and Jussi Holopainen. Patterns in Game Design. Boston, MA: Charles River Media, 2006.

7. Björk Staffan, and Jussi Holopainen, and Sus Lundgren. “Game Design Patterns." In Game Design Patterns, 2003. https://www.researchgate.net/publication/221217599_Game_Design_Patterns.

8. Butler, Tom. "The Rise of the Jump." Polygon. January 20, 2014. Accessed April 20, 2020. https://www.polygon.com/features/2014/1/20/5227582/the-rise-of-the-jump.

9. "Call of Duty 4: Modern Warfare." Pattern Language for Game Design - Games Reference. 2020. Accessed July 16, 2021. https://patternlanguageforgamedesign.com/PatternLibraryApp/GamesReference/95.

10. Dillon, Roberto. "The 6-11 Framework: A New Methodology For Game Analysis And Design." Proceedings of Game-On Asia Conference. 2011. Accessed July 16, 2021. https://www.academia.edu/1571687/THE_6_11_FRAMEWORK_A_NEW_METHODOLOGY_FOR_GAME_ANALYSIS_AND_DESIGN.

11. Eckstein, Jutta, Joseph Bergin, Mary-Lynn Manns, Helen Sharp, Markus Voelter, Eugene Wallingford, Klaus Marquardt, Jane Chandler, and Astrid Fricke. "The Pedagogical Patterns Project." The Pedagogical Patterns Project. 2012. Accessed July 16, 2021. http://www.pedagogicalpatterns.org/.

12. Flores, Nuno, Ana C. Paiva, and Nuno Cruz. "Teaching Software Engineering Topics Through Pedagogical Game Design Patterns: An Empirical Study." Information 11, no. 3 (2020): 153. doi:10.3390/info11030153.

13. "Fortnight: Battle Royal." Pattern Language for Game Design - Games Reference. 2020. Accessed July 16, 2021. https://patternlanguageforgamedesign.com/PatternLibraryApp/GamesReference/58.

14. "Game Degrees Campus \& Online - Full Sail University." Campus \& Online - Full Sail University. 2021. Accessed July 16, 2021. https://hello.fullsail.edu/brand_1ar_games.

15. "Game Science and Design, MS." Northeastern CAMD. April 06, 2021. Accessed July 16, 2021. https://camd.northeastern.edu/program/game-science-and-design-ms/.

16. Gamma, Erich, Richard Helm, Ralph E. Johnson, and John Vlissides. Design Patterns: Elements of Reusable Object-oriented Software. New Dehli: Pearson Education, 1994. 
17. Hunicke, Robin, Marc LeBlanc, and Robert Zubek. "MDA: A Formal Approach to Game Design and Game Research." Game Developers Conference, 2001.

18. Iba, Takashi. "Designing a Pattern Language for Creative Learners." MIT Center for Collective Intelligence, July 2010.

19. Kelle, Sebastian \& Klemke, Roland \& Specht, Marcus. (2011). Design Patterns for Learning Games. International Journal of Technology Enhanced Learning. 3. 10.1504/IJTEL.2011.045452.

20. Lazzaro, Nicole. "Four Keys to Fun." XEODesign®. 2015. Accessed July 16, 2021. http://www.xeodesign.com/research/.

21. Lee, Chris. "GDPVis." GDPVis. 2021. Accessed July 16, 2021. http://gdpv.is/.

22. Lindley, Craig. "Game Taxonomies: A High Level Framework for Game Analysis and Design." Gamasutra. October 3, 2003. Accessed July 16, 2021. https://www.gamasutra.com/view/feature/131205/game_taxonomies_a_high_level_.php.

23. Martin, Fasterholdt, Pichlmair Martin, and Holmgård Christoffer. "You Say Jump, I Say How High? Operationalising the Game Feel of Jumping." DiGRA. January 01, 1970. Accessed April 20, 2020. http://www.digra.org/digital-library/publications/you-say-jump-i-say-how-high-operationalising-the-game-feel-of-jumping/.

24. The Pedagogical Patterns Project (2009) Available at http://www.pedagogicalpatterns.org/ (accessed on 20 March 2009).

25. Romero, Brenda. "Train." Brenda Romero's Train. 2009. Accessed July 16, 2021. http://brenda.games/train.

26. Schell, Jesse. The Art of Game Design: A Book of Lenses. Boca Raton: CRC Press, Taylor \& Francis Group, 2020.

27. Shuell, T.J. and Moran, K.A. (1994) ‘Learning theories: historical overview and trends', in T. Husen and T.N. Postlethwaite (Eds.): The International Encyclopedia of Education, 2nd ed., pp.3340-3345, Pergamon, Oxford, UK.

28. "Spec Ops: The Line." Pattern Language for Game Design - Games Reference. 2020. Accessed July 16, 2021. https://patternlanguageforgamedesign.com/PatternLibraryApp/GamesReference/101.

29. Takashi Iba, Creative Learning Paper: https://www.researchgate.net/publication/229037946_Designing_a_Pattern_Language_for_Creative_Learners

30. VandenBerghe, Jason. "The Five Domains Of Play: Mapping Psychology's Five Factor Model to Game Design." Darklorde.com. April 06, 2017. Accessed July 16, 2021. https://www.darklorde.com/the-five-domains-of-play/.

31. "This War of Mine." Pattern Language for Game Design - Games Reference. 2020. Accessed July 16, 2021. https://patternlanguageforgamedesign.com/PatternLibraryApp/GamesReference/280.

32. "What Game Do You Think Has Perfect Jumping Mechanics?" GameFAQs. February 02, 2018. Accessed April $20,2020$. https://gamefaqs.gamespot.com/boards/204-classic-gaming/76251195.

33. "What Was the First Game with a Double Jump and Why Was It Implemented?" Reddit. 2016. Accessed April $20,2020$. https://www.reddit.com/r/truegaming/comments/4266et/what_was_the_first_game_with_a_double_jump_and/.

34. The World Wide Web Consortium. (W3C) "OWL 2 Web Ontology Language Primer (Second Edition)." OWL 2 Web Ontology Language. December 11, 2012. Accessed July 16, 2021. https://www.w3.org/TR/2012/REC-owl2-primer20121211/\#ref-owl-2-quick-reference.

\section{Reviewer Suggestions}

1. Jussi Holopainen

2. Staffan Bjork

3. Jesse Schell

4. Ernest Adams

5. Joris Dormans

6. Chris Totten 